\title{
Regeneration barrieregeschädigter Haut durch die Behandlung mit zwei Hautpflegeprodukten
}

\section{H. Löffler ${ }^{1}$ \\ H. F. Wesp ${ }^{2}$}

Regeneration of Barrier-Disrupted Skin due to Different Skin Care Products

\section{Zusammenfassung}

Irritative Schädigungen der Haut können zu komplizierenden Verläufen bei Patienten mit einer atopischen Dermatitis und mit Handekzemen führen. Die Behandlung solcher meist subklinischen Irritationen mit einer suffizienten Hautpflege führt nicht immer zu einer Besserung und ist abhängig von der Wirksamkeit des jeweiligen Hautpflegeproduktes. In der vorliegenden Studie wurden die beiden Hautpflegepräparate Menalind derm ${ }^{\circledR}$ und Eucerin für empfindliche Haut ${ }^{\circledR}$ auf ihre Eignung untersucht, nach einer subklinischen Schädigung die Barriereregeneration der Haut zur fördern und den kutanen Hydratationsgehalt zu erhöhen. 50 hautgesunde Probanden führten 7 Tage lang zweimal täglich eine Waschprozedur mit 0,5\%iger Natriumlaurylsulfat-Lösung an beiden Unterarmen durch. Im Anschluss wurde eine Hälfte des Unterarms über 10 Tage mit der einen Creme behandelt, die andere Hälfte diente als unbehandelte Kontrolle. Der zweite Unterarm wurde in gleicher Weise mit dem anderen Hautpflegeprodukt behandelt. Der Zustand der Haut wurde vor der Waschphase, zu Beginn und während der Cremephase (Tag 7,10,14 und 17) sowie einige Tage nach Beendigung (Tag 21) durch Messung des transepidermalen Wasserverlustes (TEWL) und des kutanen Hydratationsgehaltes evaluiert. Es zeigt sich, dass im Vergleich zu den Kontrollstellen beide Cremes die Regeneration der Barrierefunktion förderten (Menalind derm signifikant von Tag 14 bis 21) und den Feuchtigkeitsgehalt der Haut signifikant steigerten. Die Probanden empfanden bei beiden Hautpflegepräparaten eine deutliche Minderung des Trockenheitsgefühls. Da subklinische Irritationen den klinischen Befund von Patienten mit einer atopischen Dermatitis oder Handekzemen verschlimmern können, lassen diese Ergebnisse erwarten, dass eine Basispflege mit den getesteten Externa das klinische Bild dieser Erkrankung verbessert.

\section{Abstract}

Additional irritation may complicate the clinical course of patients with atopic dermatitis or hand eczema. The treatment of this mostly subclinical irritation with skin care products is not guaranteed and dependent on the efficacy of each product. In the present study, two skin care products (Menalind derm ${ }^{\circledR}$ und Eucerin für empfindliche Haut ${ }^{\circledR}$ ) are tested regarding their ability to enhance regeneration of a disrupted barrier and improvement of cutaneous hydration after a subclinical irritation. For seven days, 50 healthy volunteers performed a wash procedure with $0,5 \%$ sodium lauryl sulphate on both forearms twice daily. After these seven days, half of the forearm was treated with one product, the other half served as control. The second forearm was treated likewise with the other product. Skin condition was evaluated before irritation, before and during treatment (day 7,10,14 and 17) and several days after treatment (day 21) using measurement of transepidermal water loss (TEWL) and cutaneous hydration. Both products were able to enhance regeneration compared to their untreated control sides. The sensation of dryness was likewise reduced by both products. Because subclinical irritation may worsen the clinical course of atopic dermatitis or hand eczema, the results of this study lead to the assumption that skin care with the tested products may improve the course of these disorders.

${ }^{1}$ Klinik für Dermatologie und Allergologie, Philipps-Universität Marburg

${ }^{2}$ Paul Hartmann AG, Abteilung Klinische Forschung, Heidenheim

Korrespondenzadiresse

Priv.-Doz. Dr. med. H. Löffler · Dermatologische Klinik · Klinikum der Philipps-Universität · Deutschhausstr. 9 . 35037 Marburg/Lahn · E-mail: Harald.Loeffler@mailer.uni-marburg.de

Bibliografie

Akt Dermatol 2004; 30: 162-166 @ Georg Thieme Verlag KG Stuttgart · New York · DOI 10.1055/s-2004-814508

ISSN 0340-2541 
Das Erkrankungsbild der irritativen Kontaktdermatitis ist jedem Dermatologen aus seiner Praxis bekannt, zumeist als klassisches Handekzem [1]. Doch wird der Einfluss irritativer Noxen auf eine Vielzahl von Erkrankungen (Windeldermatitis, Gesichtsekzeme und insbesondere der atopischen Dermatitis) vielfach unterschätzt. Gerade bei Patienten mit einer atopischen Dermatitis, deren Haut hochgradig empfindlich auf verschiedene Alltagseinflüsse reagiert, spielt eine Irritation der Haut eine wichtige Rolle als Auslöser und Verstärker der Erkrankung [2,3]. Eine suffiziente Prophylaxe und Therapie von Hautirritationen sind daher für die Risikogruppe der Atopiker wichtig. Doch sind nicht alle Hautpflegeprodukte in der Lage, die Barrierefunktion der Haut wiederherzustellen. Von Menalind derm ${ }^{\circledR}$ ist bekannt, dass es sowohl bei Atopikern als auch bei Patienten mit trockener Haut die Barrierefunktion verbessert [4]. Manche Cremes hingegen haben nur geringe Effekte auf die irritierte Haut [5-7]. Von anderen Hautpflegeprodukten ist bekannt, dass sie eine Irritation sogar noch verstärken [8]. Anhand klinisch kontrollierter Studien sollte daher die Wirksamkeit für Hautpflegeprodukte nachgewiesen werden, die den Anspruch erheben, die Regeneration irritativ geschädigter Haut zu fördern.

In der nachfolgend beschriebenen Studie wurde in einem praxisnahen Testdesign (dem repetitiven Waschtest [9]) untersucht, ob die zwei Hautpflegeprodukte Menalind derm ${ }^{\circledR}$ und Eucerin für trockene Haut ${ }^{\circledR}$ die Regeneration geschädigter Haut unterstützen. Die beiden Cremes wurden dabei in einem Halbseitenversuch [10] verglichen.

\section{Materialien und Methoden}

\section{Probanden}

An der Studie nahmen 50 hautgesunde Probanden teil. Die Studie hatte von der zuständigen Ethik-Kommission ein positives Votum erhalten. Die Probanden wurden vor Beginn der Studie über das Studiendesign mit den möglichen Risiken und Nebenwirkungen aufgeklärt und gaben schriftlich ihr Einverständnis. Die beiden Hautpflegeprodukte Menalind $\operatorname{derm}^{\circledR}$ und Eucerin für trockene Haut ${ }^{\circledR}$ wurden auf einer experimentell irritierten Haut der Probanden (Waschtest über eine Woche mit einem Detergens: Natriumlaurylsulfat (NLS) 0,5\%) aufgetragen. Über insgesamt 21 Tage wurde dann die Regeneration der Haut beobachtet.

\section{Standardisierter Waschtest}

Der Waschtest wurde über eine Woche, von Tag 0 bis Tag 6 , durchgeführt. Der Proband führte zweimal täglich an jedem Unterarm eine Waschung mit NLS $(0,5 \% \mathrm{ig})$ in $37^{\circ} \mathrm{C}$ warmen Wasser durch. Diese Waschung erfolgte durch zehnmaliges Auf- und Abrollen mit einem in der Waschlösung getränkten Schaumstoffroller. Hiervon wurden 5 Serien durchgeführt, nach jeder Serie wurde der Roller wieder neu mit der Waschlösung getränkt. Danach wurde der Unterarm unter klarem Wasser abgewaschen und vorsichtig mit Zellstoff abgetrocknet. Für jeden Waschvorgang ( = 5 Rollerserien) wurden $50 \mathrm{ml}$ der Waschlösung benötigt.

\section{Regenerationsphase - Therapiephase}

Nach der Schädigung der Haut folgte die Anwendung der Pflegecremes. Es wurden nur diejenigen Probanden in diese Phase der Studie aufgenommen, bei denen sich der transepidermale Wasserverlust (TEWL) an Tag 7 um 50\% des Basal-Wertes erhöht hatte und die Haut somit ausreichend geschädigt worden war. Der Proband trug nun ab Tag 7 bis einschließlich Tag 16 zweimal täglich eine Spatelspitze des Hautpflegemittels auf die Unterarme auf. Die Zuordnung eines Hautpflegemittels zu einem Unterarm erfolgte randomisiert, ebenso die Zuordnung zu der Therapiefläche (distaler oder proximaler Unterarm, jeweils die andere Fläche diente als unbehandelte Kontrollfläche). Sämtliche Hautpflegemittel wurden verblindet und kostenfrei zur Verfügung gestellt. Ab Tag 17 erfolgte keine Behandlung mehr, die Zeit bis Tag 21 diente der Kontrolle nach Absetzen der Therapie.

\section{Hautphysiologische Messmethoden}

Für die Evaluation der Irritationen und der Hautregeneration wurden an Tag 0, 7, 10, 14, 17 und 21 folgende hautphysiologische Messmethoden durchgeführt:

Barriereschädigung

- Messung des transepidermalen Wasserverlustes mit dem TEWAMETER TM 210 der Firma Courage \& Khazaka, Köln.

Veränderung des epikutanen Wassergehaltes (Feuchtigkeit)

- Messung des oberflächlichen elektrischen Widerstandes mit einem Corneometer CM 829 der Firma Courage \& Khazaka, Köln

Der transepidermale Wasserverlust sowie der epikutane Feuchtigkeitsgehalt wurden an jeder Unterarm-Beugeseite im distalen und im proximalen Bereich (Therapie- und Kontrollfläche) gemessen.

\section{Verwendete subjektive Irritationsskalen}

Für die Evaluierung subjektiver Veränderungen der Haut wurde bei den Waschtests ein Irritationsscore erhoben. Dabei erfolgte eine Unterscheidung bezüglich der am häufigsten genannten Probleme Trockenheit, Juckreiz und Brennen. Die Parameter wurden in einer Skala von 0-10 (keine Symptome - maximal starke Symptome) an den Auswertungstagen (Tag 0, 7, 10, 14, 17 und 21) abgefragt. Die Fragebogen basieren auf den „Guidelines on sodium lauryl sulfate (SLS) exposure testing“ der „Standardisation Group of the European Society of Contact Dermatitis“[11].

\section{Statistik}

Zunächst wurde die Normalverteilung aller Messdaten mit dem Kolmogorov-Smirnov-Test überprüft. Es zeigt sich durchweg eine Normalverteilung. Der Vergleich von Messwerten wurde daraufhin mit dem t-Test durchgeführt. Die subjektiven Symptomscores folgten keiner Normalverteilung. Der Vergleich der Mediane wurde daraufhin mit dem Wilcoxon-Test vorgenommen. 
Tab. 1 a Reduktion der $\Delta$-TEWL-Werte, ausgehend vom maximalen Irritationswert an Tag 7

\begin{tabular}{|lcccc}
\hline & Menalind derm & Kontrolle & Eucerin & Kontrolle \\
\hline Tag 7 & 0 & 0 & 0 & 0 \\
\hline Tag 10 & $-9,2 \pm 8,4$ & $-7,4 \pm 6,3$ & $-8,9 \pm 8,6$ & $-7,7 \pm 7,2$ \\
\hline Tag 14 & $-13,5 \pm 9,2$ & $-10,7 \pm 7,4$ & $-13,1 \pm 10,6$ & $-12,0 \pm 8,4$ \\
\hline Tag 17 & $-15,6 \pm 9,4$ & $-12,4 \pm 7,6$ & $-15,3 \pm 10,8$ & $-13,2 \pm 8,4$ \\
\hline Tag 21 & $-17,1 \pm 10,3$ & $-14,1 \pm 8,0$ & $-16,2 \pm 10,5$ & $-15,2 \pm 9,3$ \\
\hline
\end{tabular}

Tab. 1 b Anstieg der $\Delta$-Corneometrie-Werte, ausgehend vom maximalen Irritationswert an Tag 7

\begin{tabular}{|ccccc|}
\hline & Menalind derm & Kontrolle & Eucerin & Kontrolle \\
\hline Tag 7 & 0 & 0 & 0 & 0 \\
\hline Tag 10 & $6,6 \pm 12,5$ & $-3,4 \pm 8,2$ & $8,7 \pm 14,3$ & $-1,6 \pm 10,8$ \\
\hline Tag 14 & $13,1 \pm 11,1$ & $1,3 \pm 11,7$ & $13,0 \pm 12,1$ & $3,2 \pm 13,0$ \\
\hline Tag 17 & $13,1 \pm 10,2$ & $5,2 \pm 11,4$ & $15,2 \pm 11,0$ & $7,0 \pm 12,8$ \\
\hline Tag 21 & $9,3 \pm 12,7$ & $8,3 \pm 10,6$ & $10,8 \pm 11,3$ & $9,3 \pm 13,4$ \\
\hline
\end{tabular}

Ergebnisse

Die Messergebnisse als Delta-Werte (Unterschied zum maximalen Irritationswert an Tag 7, mit Normierung auf den Basalwert) finden sich in Tab.1 a,b.

\section{Entwicklung des transepidermalen Wasserverlustes}

Durch die Waschung kam es zu einer deutlichen Erhöhung des TEWL, welche die Barriereschädigung der Haut nachweisen konnte. Im Verlauf der TEWL-Werte nach Beendigung der Waschphase und gleichzeitigem Beginn der Therapiephase (ab Tag 7) kam es sowohl bei den behandelten als auch den unbehandelten Hautarealen zu einem Abfall der TEWL-Werte (Abb.1). Dieser Abfall war ausgeprägter für die behandelten Areale als für die Kontrollstellen und überdauerte die Phase des Eincremens (Tag 7 - 16). Der Unterschied zwischen der TEWL-Reduktion durch Menalind derm ${ }^{\circledR}$ im Vergleich zu seiner Kontrollstelle war an den Tagen $14-21$ signifikant $(p<0,05)$. Eucerin für empfindliche Haut ${ }^{\circledR}$ verfehlte die Signifikanzschwelle.

\section{Entwicklung des kutanen Feuchtigkeitsgehaltes}

Durch die Waschung der Haut fielen die Corneometie-Werte zunächst deutlich, da die Hautoberflächenfeuchtigkeit abnahm. Auch nach Beendigung der austrocknenden Waschphase verringerten sich die Corneometrie-Werte ohne weitere Behandlung zunächst weiter (bis Tag 10), bevor sie dann mit Tag 21 wieder den Ausgangswert erreichten (Abb.2). Bei den Therapiestellen zeigte sich ein deutlicher Anstieg bereits kurz nach Beginn der Behandlung (Tag 10), welcher anhielt bis an Tag 17. Nach Beendigung der Therapiephase (ab Tag 17) kam es wieder zu einer Abnahme bis auf die Ausgangswerte. Die Corneometrie-Werte beider Hautpflegeprodukte waren an den Tagen 10-17 signifikant höher als die der Kontrollstellen $(\mathrm{p}<0,001)$.

\section{Mittelwerte der subjektiven Beurteilung}

Subjektive Beschwerden äußerten die Probanden vor allem an den Tagen 7-14 (Tab.2). Während bei den Kriterien "Juckreiz“ und „Brennen“ nur ein geringer Unterschied zwischen den beiden Hautpflegeprodukten und den Kontrollstellen zu erkennen war, so wurde bei dem Kriterium „Trockenheit“ der Unterschied deutlich: Die Probanden empfanden die eingecremte Haut als wesentlich weniger trocken. Der Unterschied zu der jeweiligen Kontrollstelle war für Menalind derm ${ }^{\circledR}$ dabei an allen Tagen signifikant, bei Eucerin für empfindliche Haut ${ }^{\circledR}$ an den Tagen 10 - 17 .

\section{Diskussion}

Eine trockene, irritativ geschädigte Haut gilt immer noch bei vielen Ärzten als ein vorwiegend kosmetisches Problem und wird kaum beachtet. Dabei darf man diesen Zustand der Haut nicht gleichsetzen mit einer empfindlichen Haut, da der Begriff der Empfindlichkeit durch seine unscharfe Definition und seinen Missbrauch als eine „Life-style“-Erkrankung gekennzeichnet ist [12]. Das Problem der irritativ vorgeschädigten Haut hingegen selbst wenn diese Schädigung nur subklinisch ist - ist vor allem bei berufsdermatologischen Patienten und Atopikern von hoher Relevanz [2,3]. So konnte gezeigt werden, dass die irritative Vorschädigung der atopischen Haut zu einer deutlichen Verstärkung der Reaktion auf Aeroallergene führt [13]. Dass eine subklinische Irritation weitere irritative Einflüsse verstärkt, ist mittlerweile unumstritten $[14,15]$. Prophylaktisch wie therapeutisch ist daher die Unterstützung der Regeneration trockener, irritierter Haut wichtig. Doch ist nicht jedes Hautpflegepräparat in der Lage, die Regeneration zu unterstützen. Manche Präparate verzögern die Regeneration der Barrierefunktion der Haut sogar [8]. Eine Prüfung für jedes Präparat ist daher zu fordern.

In der vorliegenden Studie konnte bei beiden getesteten Externa eine Unterstützung der Hautregeneration gesehen werden. Zunächst stellten beide Pflegepräparate die Barrierefunktion der Haut deutlich schneller wieder her, gemessen am Rückgang des transepidermalen Wasserverlustes. Menalind derm ${ }^{\circledR}$ verringerte dabei - im Vergleich zur unbehandelten Haut - den TEWL signifikant an den Tagen 14-21.

Dass diese Beobachtung nicht auf einen einfachen okkludierenden Effekt zurückgeführt werden kann, zeigt der Kurvenverlauf des TEWL, der eine Zunahme des Effektes (im Vergleich zur unbehandelten Kontrolle) über die Behandlungszeit aufweist. Dieser Unterschied zur Kontrolle war für Menalind derm ${ }^{\circledR}$ zunehmend vom 14. Tag bis zum Ende der Beobachtungszeit (Tag 21) signifikant. Im Gegensatz zu diesem Verlauf müsste bei einer einfachen Okklusion der Unterschied zur unbehandelten Kontrolle direkt nach der Behandlung am größten sein und mit der Zeit dann wieder abnehmen. Betrachtet man sich außerdem den Verlauf des TEWL nach Beendigung der Eincremephase (ab Tag 17), müsste der TEWL bei einem reinen Okklusionseffekt wieder ansteigen. Hier war das Gegenteil der Fall: Auch nach Beendigung der Therapiephase reduzierte sich der TEWL weiter. Dies zeigt, dass die Barriereregeneration weiter fortschreitet und nicht nur auf die Therapiephase begrenzt ist. Durch die Therapie konnte die Regeneration jedoch auf einer schon besseren Hautqualität fortfahren. Aufgrund dieser Beobachtungen kann daher 

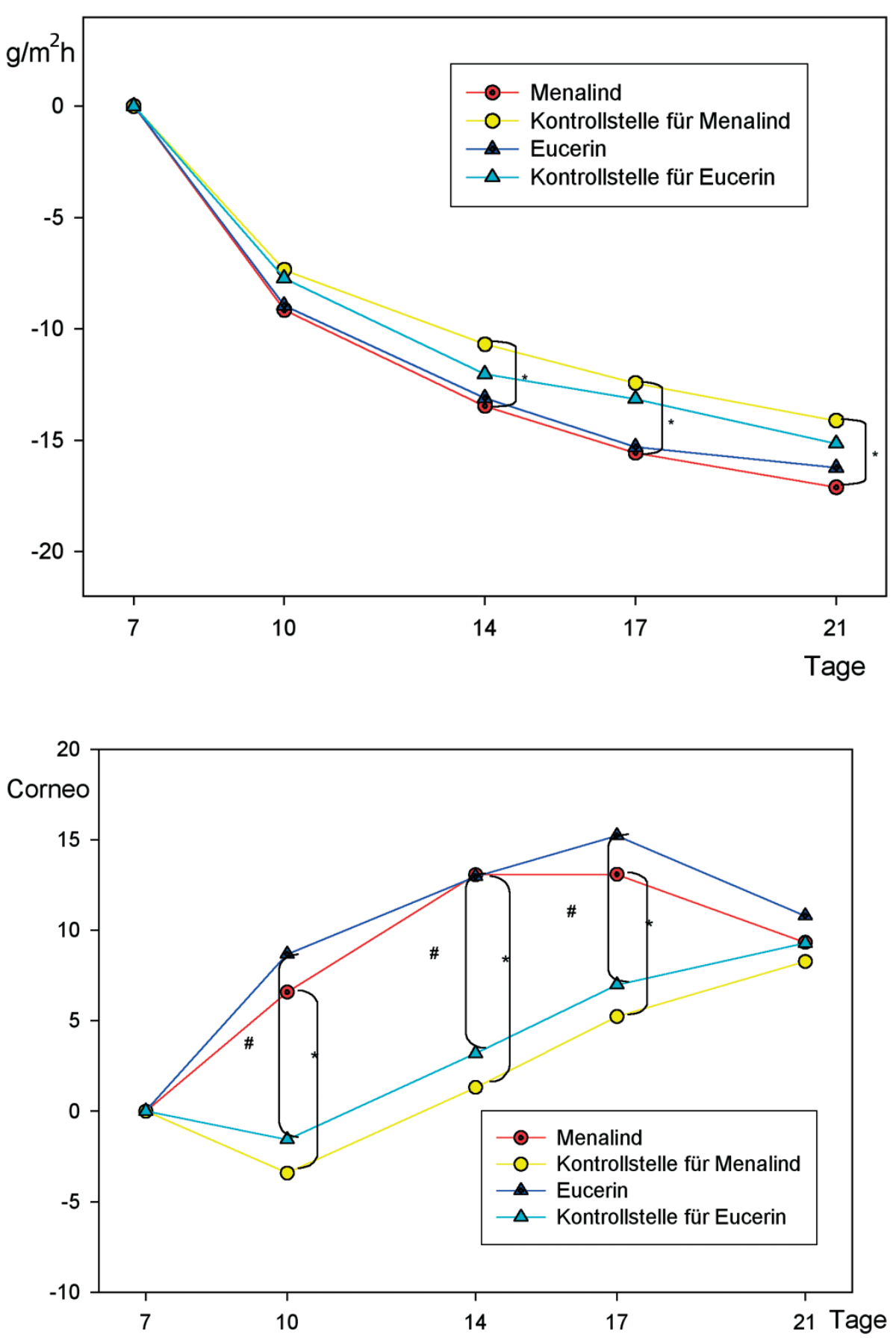

Abb. 1 Entwicklung des transepidermalen Wasserverlustes nach Abschluss der Waschphase. *: Unterschied zwischen Menalind derm und seiner Kontrollstelle ist signifikant $(p<0,05)$.

Abb. 2 Corneometrie-Entwicklung nach Abschluss der Waschphase ( ${ }^{*}$ und ${ }^{\#}$ : Unterschied zur Kontrollstelle ist signifikant $[p<0,001])$.

Tab. 2 Mittelwerte der subjektiven Beurteilung

\begin{tabular}{|c|c|c|c|c|c|c|c|c|c|c|c|c|}
\hline Tag & $\begin{array}{l}\text { Juckreiz } \\
\text { Menalind }\end{array}$ & $\begin{array}{l}\text { Kontrolle } \\
\text { Menalind }\end{array}$ & Eucerin & $\begin{array}{l}\text { Kontrolle } \\
\text { Eucerin }\end{array}$ & $\begin{array}{l}\text { Trockenheit } \\
\text { Menalind }\end{array}$ & $\begin{array}{l}\text { Kontrolle } \\
\text { Menalind }\end{array}$ & Eucerin & $\begin{array}{l}\text { Kontrolle } \\
\text { Eucerin }\end{array}$ & $\begin{array}{l}\text { Brennen } \\
\text { Menalind }\end{array}$ & $\begin{array}{l}\text { Kontrolle } \\
\text { Menalind }\end{array}$ & Eucerin & $\begin{array}{l}\text { Kontrolle } \\
\text { Eucerin }\end{array}$ \\
\hline 0 & 0 & 0 & 0 & 0 & 0 & 0 & 0 & 0 & 0 & 0 & 0 & 0 \\
\hline 7 & 2,7 & 2,8 & 2,6 & 2,9 & 4,4 & 4,5 & 4,4 & 4,4 & 2,4 & 2,4 & 2,8 & 2,8 \\
\hline 10 & 1,4 & 1,5 & 1,0 & 1,6 & 1,7 & 2,5 & 1,4 & 2,6 & 0,8 & 0,6 & 0,6 & 0,6 \\
\hline 14 & 0,4 & 0,4 & 0,3 & 0,4 & 0,4 & 1,4 & 0,5 & 1,4 & 0,2 & 0,1 & 0,2 & 0,2 \\
\hline 17 & 0 & 0 & 0,1 & 0,1 & 0,2 & 0,6 & 0 & 0,5 & 0 & 0 & 0 & 0 \\
\hline 21 & 0 & 0 & 0,2 & 0,2 & 0 & 0,2 & 0,2 & 0,2 & 0 & 0 & 0,2 & 0,2 \\
\hline
\end{tabular}


angenommen werden, dass die Pflegecremes über ihre physikalische Wirkung hinaus eine tatsächliche Förderung der Barriereregeneration induzieren.

Der eindrucksvollste Effekt durch Pflegecremes lässt sich jedoch an der Zunahme der Hautoberflächenfeuchtigkeit (gemessen durch die Corneometrie) erkennen. Zunächst kam es durch die Waschungen zu einer deutlichen Abnahme der Hautoberflächenfeuchtigkeit. Diese Beobachtung ist für die Irritation mit NLS bekannt und simuliert eine Vorschädigung der Haut, wie sie bei Feuchtarbeit und häufigem Waschen vorkommt [16-18]. Makroskopisch war diese Schädigung durch eine feinlammelläre Schuppung der Haut an den Unterarmen zu erkennen. Nach dem Auftragen der Pflegecremes stieg die Hautoberflächenfeuchtigkeit deutlich an, induziert durch die Applikation von wasserbindenden Substanzen und Lipiden. Der Unterschied zu den unbehandelten Kontrollen war dabei zu jeder Behandlungszeit signifikant. Interessant ist die deutliche Abnahme der Hautoberflächenfeuchtigkeit nach Beendigung der Cremephase (innerhalb von 5 Tagen fast bis auf das Niveau der Kontrollstelle). Dies zeigt auf, dass der Hydratisierungs-Effekt nur kurzfristig anhält und nach Absetzen der Creme auch rasch wieder zurückgeht. In der Konsequenz bedeutet dies, dass der Patient regelmäßig behandelt werden sollte, um den gesteigerten Hydratisierungszustand der Haut aufrecht zu erhalten.

Die Behandlung selbst wurde von den Probanden als angenehm empfunden. Während der Juckreiz und das durch die Irritation verursachte Brennen kaum beeinflusst wurden, konnte das Trockenheitsgefühl durch das Eincremen wirksam gemindert werden.

Die regelmäßige Hautpflege zählt seit langem zum Standard der Behandlung von Patienten mit einer atopischen Dermatitis. Unsere Untersuchungsergebnisse bestätigen, dass die Hautpflege mit Menalind derm und Eucerin die Regeneration irritativ geschädigter Haut - von der Atopiker häufig betroffen sind - unterstützt, die Hydratation erhöht und die subjektiven Beschwerden mindert. Dadurch sollte es gelingen, allein schon mit einer guten Basispflege die subklinische Schädigung der atopischen Haut zu minimieren, um so additiven Schäden (durch exogene Allergene, Irritantien, aber auch durch endogene Schübe) vorzubeugen $[15,19]$.

\section{Literatur}

${ }^{1}$ Löffler H, Effendy I, Happle R. Die irritative Kontaktdermatitis. Hautarzt 2000; 51: $203-215$

${ }^{2}$ Halkier-Sörensen L. Occupational skin diseases. Contact Dermatitis 1996; 35 Suppl1: $1-120$

${ }^{3}$ Nassif A, Chan SC, Storrs FJ, Hanifin JM. Abnormal skin irritancy in atopic dermatitis and in atopy without dermatitis. Arch Dermatol 1994; 130: $1402-1407$

${ }^{4}$ Bielfeld S, Wigger-Alberti W, Wesp HF. Behandlung trockener Hautzustände bei Hautgesunden und Personen mit atopischer Diathese. Akt Dermatol 2002; 28: 443-448

${ }^{5}$ Frosch PJ, Kurte A, Pilz B. Efficacy of skin barrier creams (III): The repetitive irritation test (RIT) in humans. Contact Dermatitis 1993; 29: $113-118$

${ }^{6}$ Frosch PJ, Schulze DirksA, Hoffmann M, Axthelm I. Efficacy of skin barrier creams (II): Ineffectiveness of a popular "skin protector" against various irritants in the repetitive irritation test in the guinea pig. Contact Dermatitis 1993; 29: 74-77

${ }^{7}$ Frosch PJ, Kurte A. Efficacy of skin barrier creams (IV): The repetitive irritation test (RIT) with a set of 4 standard irritants. Contact Dermatitis $1994 ; 31: 161-168$

${ }^{8}$ Held E, Sveinsdottir S, Agner T. Effect of long-term use of moisturizer on skin hydration, barrier function and susceptibility to irritants. Acta Derm Venereol 1999; 79: 49-51

${ }^{9}$ Gehring W, Gloor M. Der repetitive Waschtest als Modell zur Beurteilung von Hautschutzpräparaten am Beispiel einer dexpanthenolhaltigen Formulierung. Akt Dermatol 2001; 27: 279-284

${ }^{10}$ Löffler H, Effendy I. Hautschutz oder Hautregenerationscreme? Der Halbseitenversuch in der Bewertung eines hautpflegenden Externums. Z Hautkr 2002; 44: 234-238

11 Tupker RA, Willis C, Berardesca E, Lee SH, Fartasch M, Agner T, Serup J. Guidelines on sodium lauryl sulfate (SLS) exposure testing. Contact Dermatitis 1997; 37: 53-69

${ }^{12}$ Löffler H, Dickel H, Kuss O, Diepgen TL, Effendy I. Characteristics of self-estimated enhanced skin susceptibility. Acta Derm Venereol $2001 ; 81: 343-346$

${ }^{13}$ Löffler H, Steffes A, Happle R, Effendy I. Allergy and irritation: an adverse association in patients with atopic eczema. Acta Derm Venereol 2003; 83: 328 - 331

${ }^{14}$ Löffler H, Effendy I, Happle R. Irritant contact dermatitis. Hautarzt 2000; 51: 203-215

${ }^{15}$ Willis CM. Variability in responsiveness to irritants: thoughts on possible underlying mechanisms. Contact Dermatitis 2002; 47: 267-271

${ }^{16}$ Grunewald AM, Gloor M, Gehring W, Kleesz P. Damage to the skin by repetitive washing. Contact Dermatitis 1995; 32: 225 - 232

17 Klein G, Grubauer G, Fritsch P. The influence of daily dish-washing with synthetic detergent on human skin. Br J Dermatol 1992; 127: $131-137$

${ }^{18}$ Malten KE. Thoughts on irritant contact dermatitis. Br J Dermatol 1981; 7: $238-247$

${ }^{19}$ Tupker RA, Coenraads PJ, Fidler V, de Jong MC, van der Meer JB, de Monchy JG. Irritant susceptibility and weal and flare reactions to bioactive agents in atopic dermatitis: I. Influence of disease severity. $\mathrm{Br} \mathrm{J}$ Dermatol 1995; 133: $358-364$ 